\title{
Toraks cerrahisinde postoperatif analjezi yönetimi: iki yıllık deneyimlerimiz
}

\section{Postoperative analgesia management in thoracic surgery: our two-year experience}

\author{
Gülay ÜLGER* $⿴$, Musa ZENGIN⿴, Ramazan BALDEMiR
}

Ankara Göğüs Hastalıkları ve Göğüs Cerrahisi Eğitim ve Araştırma Hastanesi, Anestezi ve Reanimasyon Kliniği, Ankara/TÜRKiYE

\section{öz}

Amaç: Toraks cerrahisi, en ağrılı cerrahi prosedürlerden biri olarak kabul görmektedir. Toraks cerrahisinden sonra gelişen ağrl; atelektazi ve pnömoni gibi solunum komplikasyonları, daha uzun hastane yatış süreleri, yaşam kalitesinin azalması ve ağrının postoperatif kronik ağrıya dönüşmesi gibi olumsuz sonuçlara neden olabilir. Toraks cerrahisi geçiren hastalarda postoperatif analjezi uygulamalarımızı ve sonuçlarını retrospektif olarak incelemeyi amaçladık.

Gereç ve Yöntemler: Çalışmamız, Haziran 2019 ve Haziran 2021 tarihleri arasında toraks cerrahisi geçiren ve postoperatif analjezi takibi yapılan 563 hastaya ait verinin retrospektif olarak incelenmesi ile yapılmıştır. Hastalara uygulanan postoperatif analjezi yöntemi, vizüel analog skala (VAS) skorları, uygulanan ek analjezi tedavisi ve gelişen komplikasyonlar değerlendirilmiştir.

Bulgular: Hastaların \%28,42'si kadın olup, en sık uygulanan cerrahi \%64,30 oranla akciğer rezeksiyonlarıydı. Hasta kontrollü analjezi (HKA), 458 (\%81,35) hastada torasik paravertebral blok (TPVB) + intravenöz, 105 (\%18,65) hastada epidural yol ile uygulanmıştı. TPVB + intravenöz HKA için VAS skor ortalamaları postoperatif 1. saatte 4,1; 24. saatte 2,0 ve 48. saatte 1,2 idi. Epidural HKA için ise 1.saatte 3,9; 24.saatte 2,0 ve 48.saatte 1,4 olarak değerlendirilmiştir. TPVB + intravenöz HKA uygulanan hastalarda yan etki oranları \%6,99; epidural HKA uygulanan hastalarda \%37,14 olarak değerlendirilmiştir.

Sonuç: Kliniğimizde sıklıkla malignite cerrahisi yapılmaktadır. Bu hastalarda TPVB ve intravenöz analjezi kombinasyonu ile elastomerik infüzyonla uygulanan torasik epidural analjezide sonuçların benzer olduğu görülmüştür. Komplikasyon oranları TPVB ve intravenöz analjezi kombinasyonu yapılan grupta daha az olmakla birlikte her iki grupta da klinik olarak kabul edilebilir sınırlarda bulunmuştur. Analjezi protokollerinin ve algoritmaların klinisyenlerin deneyimi ve uygulanan cerrahi işleme göre belirlenmesinin etkin analjezi sağlayacağını düşünmekteyiz.

Anahtar kelimeler: Toraks cerrahisi; postoperatif ağrl; hasta kontrollü analjezi yönetimi

Sorumlu Yazar*: Gülay Ülger, Ankara Göğüs Hastalıkları ve Göğüs Cerrahisi Eğitim ve Araştırma Hastanesi, Anestezi ve Reanimasyon Kliniği, Ankara/TÜRKiYE E-posta: gulayulger@gmail.com

ORCID: 0000-0003-1926-4770

Gönderim: 05.10.2021 kabul: 01.11.2021

Doi: $10.18663 / \mathrm{tjcl} .1004686$ 


\begin{abstract}
Aim: BThoracic surgery is one of the most painful surgical procedures. Pain after thoracic surgery can cause respiratory complications, such as atelectasis and pneumonia; longer hospital stays; reduced quality of life and chronic persistent postoperative pain. We aimed to retrospectively analyze our postoperative analgesia practices and results in patients undergoing thoracic surgery through anesthesia and pain follow-up forms.
\end{abstract}

Material and Methods: In our study, we retrospectively scanned 563 patients who underwent thoracic surgery and were followed up with postoperative analgesia between June 2019 and June 2021. Postoperative analgesia method applied to the patients, visual analog scale (VAS) scores, additional applied analgesia and complications were evaluated.

Results: $28.42 \%$ of the patients were women. The most common surgery was lung resections with a rate of $64.30 \%$. Patient-controlled analgesia (PCA) was administered thoracic paravertebral block (TPVB) + intravenously in 458 (81.35\%) patients and by epidural route in 105 (18.65\%) patients. The VAS scores were 4.1 at the postoperative 1 st hour, 1.9 at 24 th hour and 1.1 at 48th hour for TPVB + intravenous PCA. For epidural PCA, VAS scores were 3.9 at the first hour, 1.9 at 24th hour, and 1.4 at 48th hour. Side-effect rates were evaluated as $6.99 \%$ in patients who underwent TPVB + intravenous PCA and $37.14 \%$ in patients who underwent epidural PCA.

Conclusion: Malignancy surgery is frequently performed in our clinic. In these patients, similar analgesic results were obtained for the combination of TPVB and intravenous analgesia, and thoracic epidural analgesia (TEA) administered with elastomeric infusion. Although the complication rates were lower in the group treated with TPVB and intravenous analgesia, they were found to be within clinically acceptable limits in both groups. We think that the choosing analgesia protocols and algorithms according to the experience of the clinicians and the surgical procedure will provide effective analgesia.

Keywords: Thoracic surgery, postoperative pain; patient controlled analgesia management

\section{Giriş}

Toraks cerrahisi, en ağrılı cerrahi operasyonlardan biri olarak kabul görmektedir [1]. Torakoskopi tekniğinin gelişimiyle, video yardımlı göğüs cerrahisinde (VATS) kullanılan daha küçük kesiler sayesinde postoperatif ağrı azalmasına rağmen toraks cerrahisi sonrası ağrı kontrolü hala zorlayıcı bir durum olarak karşımıza çıkmaktadır. [2]. Bu cerrahilerden sonra en sık ağrı kaynağı cerrahi kesi, kot yaralanması, göğüs tüpleri ve sütur teknikleri olarak listelenebilir [1]. Bu cerrahi prosedürlerden sonra gelişen ağrı; atelektazi ve pnömoni gibi solunum komplikasyonları, daha uzun hastane yatış süreleri, yaşam kalitesinin azalması ve ağrının postoperatif kronik ağrıya dönüşmesi gibi olumsuz sonuçlara neden olabilir [1, 2]. Toraks cerrahisi geçiren hastaların çoğunda ciddi kardiyopulmoner komorbiditeler eşlik eder. Bu durum ağrının kontrol altına alınmasını zorlaştırır. Bu sebeple, efektif ve güvenilir ağrı yönetimi toraks cerrahisinin en önemli unsurlarındandır [3].

Toraks cerrahisinde sistemik ajanlar ve rejyonel anestezi gibi birçok analjezi seçeneği mevcuttur [3]. Toraks cerrahisi sonrası ağrı yönetiminde altın standart torasik epidural analjezi (TEA) olarak kabul edilir. Ancak, bu yönteme bağlı sempatik blokaj, solunum depresyonu ve üriner retansiyon gibi yan etkiler yanında epidural hematom ve abse gibi ciddi durumlarla da karşılaşılabilir [2].
Torasik paravertebral blok (TPVB); TEA'ya benzer analjezi sunmakla birlikte daha az postoperatif bulantı ve kusma, hipotansiyon ve üriner retansiyona neden olur [3]. Torasik cerrahi sonrası analjezi yönetiminde rejyonel sinir bloku kullanımının önemi de son on yılda artış göstermiştir [2, 5, 6]. Erector spina plane blok, serratus anterior plane blok ve interkostal blok bu rejyonel blok uygulamalarındandır. Bu uygulamaların yanında opioidler, parasetamol, nonsteroidal anti-inflammatuar ilaçlar gibi intravenöz analjezi tedavileri de rejyonel blok uygulamalarına ek olarak multimodal analjezinin bir komponenti şeklinde uygulanmaktadır $[7,8]$.

Biz bu çalışmamızda toraks cerrahisi geçiren hastalarda postoperatif analjezi uygulamalarımızı ve sonuçlarını anestezi ve ağı takip formları üzerinden retrospektif olarak incelemeyi amaçladık.

\section{Gereç ve Yöntemler}

Çalışma için etik kurul onayı (2012-KAEK-15/2344) alındı. Tüm hastalardan uygulanan analjezik tedavileri konusunda sözel ve yazılı onamları alınmıştır. Çalışmamız toraks cerrahisi ve göğüs hastalıkları konusunda hakem hastane olan bir merkezin, yaklaşık iki yıllık bir süre içindeki (1 Haziran 2019 - 30 Haziran 2021) verilerini kapsamaktadır. Çalışmamız için elektif göğüs cerrahisi girişimlerinde anestezistler tarafından postoperatif 
analjezi amacıyla epidural HKA, intravenöz HKA ve TPVB uygulanan erişkin hastaların kayıtlı dosya bilgileri incelenmiştir.

18 - 80 yaş arası, Amerikan Anestezistler Derneği (ASA) fiziksel durumu 1-3 olan, elektif göğüs cerrahisi ameliyatı geçiren, postoperatif analjezi takibi yapılan hastalar çalışmamıza dâhil edilmiştir. 18 yaş altı - 80 yaş üstü, acil şartlarda ameliyata alınmış, göğüs cerrahisi ameliyatı geçirmeyen, kronik ağrı tedavisi gören ve araştırma ile ilgili verileri eksik olan hastalar çalışma dışında bırakılmıştır.

Kliniğimizde, postoperatif analjezi takibi için kullanılan standart bir form bulunmaktadır. Bu formda demografik bilgiler (ad-soyad, yaş, cinsiyet, vücut kitle indeksi) yanında ASA fiziksel durumu, tanısı, yapılan operasyon, operasyon tipi, operasyon süresi, uygulanan postoperatif analjezi yöntemi, ağrı düzeyi değerlendirmesi, uygulanan ek analjezi tedavisi, analjezik uygulamasına bağlı yan etkiler ile hasta memnuniyeti değerlendirilmektedir. Ağrı değerlendirmesinde visüel analog skala (VAS) skoru; yan etki değerlendirmesinde baş ağrısı, bulantı-kusma, hipotansiyon, bradikardi, kaşıntı, terleme, solunum depresyonu vb. kaydedilmektedir. Hasta memnuniyeti düzeyi için ise sayısal bir ölçek (3 - çok iyi, 2 - iyi ve 1 - kötü) kullanılmaktadır.

Çalışma Hastalarındaki Torasik Paravertebral Blok + İntravenöz ve Torakal Epidural Analjezi Protokolleri

Hastalara kliniğimizde uygulanan rutin analjezi protokolüne uygun şekilde tedavi düzenlenmiştir. Preoperatif bilgilendirme sürecinde hastalar uygulanacak analjezi yöntemi ve VAS hakkında bilgilendirilmiştir. Renal ve hepatik fonksiyonlarında sorun olan, kullanılacak ilaçlara alerjisi olan ve uygulanacak analjezi yöntemini kabul etmeyen hastalara rutin klinik uygulamamız dışında farklı bir analjezik protokol uygulanmıştır. Operasyon bitmeden $20 \mathrm{dk}$. önce analjezi amaciyla $50 \mathrm{mg}$ IV deksketoprofen ve $100 \mathrm{mg}$ IV tramadol uygulanırken, antiemetik amaçlı da hastalara $10 \mathrm{mg}$ IV metoklopramid uygulanmıştır.

Postoperatif dönemde epidural analjezi yönetimi için; 270 $\mathrm{ml}$ elastomerik infüzyon pompası ile $67,5 \mathrm{ml}$ bupivakain, 201,5 ml SF ve 10 mg/ml morfin karıştırılarak elde edilen $\% 0.125$ yoğunlukta bupivakain infüzyonu postoperatif 3 gün boyunca epidural yolla elastomerik pompa ile $4 \mathrm{ml} / \mathrm{h}$ hızında başlanarak verilmiştir. Ayrıca elastomerik infüzyon pompası her 15 dakikada bir 0,5 ml bolus uygulanacak özellikte olup, hastalar bu uygulama hakkında bilgilendirilmiştir.

TEA uygulanmayan tüm hastalara cerrahi bitiminden önce lateral dekübit pozisyonda antisepsi kurallarına uyularak cildin temizlenip örtülmesinden sonra, T5 spinoz çıkıntının $2-3 \mathrm{~cm}$ lateraline $20 \mathrm{ml}$ bupivacaine kullanılarak TPVB uygulanmıştır. Bu hastalara postoperatif dönemde intravenöz hasta kontrollü analjezi (HKA) uygulanmıştır. HKA protokolü; $100 \mathrm{ml}$ izotonik sodyum klorür içine 400-500 mg tramadol eklenerek 10mg/ saat bazal infüzyon, 5-20 mg bolus, 20-30 dakika kilitli kalma süresi ve 4 saat limit 100 mg olacak şekilde uygulanmıştır.

Bütün hastalara multimodal analjezi amaçlı her 8 saatte bir parasetamol $1 \mathrm{gr}$ ve $50 \mathrm{mg}$ deksketoprofen her 12 saatte bir intravenöz verilmiş, VAS değeri 4 ve üzerinde olan hastalara "ek analjezik" olarak 50 mg intravenöz tramadol uygulanmıştır.

\section{Bulgular}

Belirtilen tarih aralığı içinde merkezimizde toplam 1624 hasta göğüs cerrahisi operasyonu geçirmiştir. İncelemeye dâhil edilmeyen hastaların özellikleri şöyledir: 18 yaş altı veya 80 yaş üstü, acil göğüs cerrahisi ameliyatı geçiren, araştırma ile ilgili verileri eksik olan. 1061 hasta çalışmaya dâhil edilmemiştir. Çalışmamız için toplam 563 hastanın verileri incelemeye dâhil edilmiştir (Şekil 1).

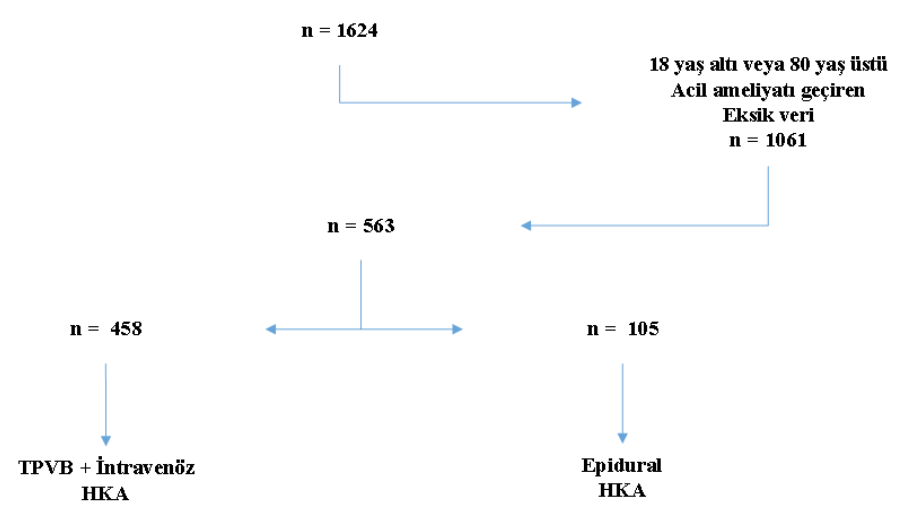

Şekil 1. Akış Şeması

Hastaların \%28,42'si kadın olup, en sık uygulanan cerrahi \%64,30 oranla akciğer rezeksiyonlarıydı. Hastaların genel özellikleri tablo 1'de özetlenmiştir (Tablo 1).

HKA, 458 (\%81,35) hastada TPVB + intravenöz, 105 (\%18,65) hastada epidural yol ile uygulanmıştı. Hastaların çoğunluğu ASA 2 ve 3 sınıfındadır (Tablo 2). Hastaların uygulanan HKA yöntemine göre memnuniyet düzeyleri ve ek analjezik ihtiyaçları da tablo 2'de gösterilmiştir (Tablo 2).

VAS skorları postoperatif 1. saatte TPVB + intravenöz HKA için 4,1 iken epidural HKA için 3,9 olarak 24. ve 48. Saatlerde TPVB + intravenöz HKA için sırasıyla 1,9 ve 1,1 iken epidural HKA için 1,9 ve 1,4 olarak değerlendirilmiştir. TPVB + İntravenöz ve Epidural HKA uygulamasında zaman içinde VAS skorlarındaki değiş̧imler şekil 2'de gösterilmiştir (Şekil 2). 


\begin{tabular}{|c|c|c|}
\hline Özellikler & $\mathbf{n}$ & $\%$ \\
\hline Toplam Hasta & 563 & 100 \\
\hline \multicolumn{3}{|l|}{ Cinsiyet } \\
\hline Kadın & 160 & 28,42 \\
\hline Erkek & 403 & 71,58 \\
\hline \multicolumn{3}{|l|}{ Yaş (yıl) } \\
\hline Kadın & $49,81 \pm 15,31$ & \\
\hline Erkek & $55,02 \pm 14,88$ & \\
\hline \multicolumn{3}{|l|}{ Tanı } \\
\hline Kitle, malignite & 422 & 74,96 \\
\hline Bronşiektazi & 27 & 4,80 \\
\hline Kist hidatik & 30 & 5,33 \\
\hline Plevral kalınlaşma, efüzyon & 48 & 8,53 \\
\hline İnterstisiyel Akciğer Hastalıkları & 5 & 0,89 \\
\hline Diğer & 31 & 5,51 \\
\hline \multicolumn{3}{|l|}{ Operasyon } \\
\hline Wedge, lobektomi, segmentektomi & 362 & 64,30 \\
\hline Pnömonektomi & 52 & 9,24 \\
\hline Eksplorasyon dekortikasyon & 53 & 9,41 \\
\hline Kistetomi & 29 & 5,15 \\
\hline Diğer & 67 & 11,90 \\
\hline \multicolumn{3}{|l|}{ Operasyon Tipi } \\
\hline VATS & 89 & 15,81 \\
\hline Torakotomi & 366 & 65,01 \\
\hline VATS + Torakotomi & 108 & 19,18 \\
\hline \multicolumn{3}{|l|}{ HKA uygulanma şekli } \\
\hline İntravenöz & 458 & 81,35 \\
\hline Kadın & 143 & 25,40 \\
\hline Erkek & 315 & 55,95 \\
\hline Epidural katater & 105 & 18,65 \\
\hline Kadın & 17 & 3,02 \\
\hline Erkek & 88 & 15,63 \\
\hline \multicolumn{3}{|c|}{ VATS: Video destekli torakoskopik cerrahi; HKA: Hasta kontrollü analjezi } \\
\hline
\end{tabular}

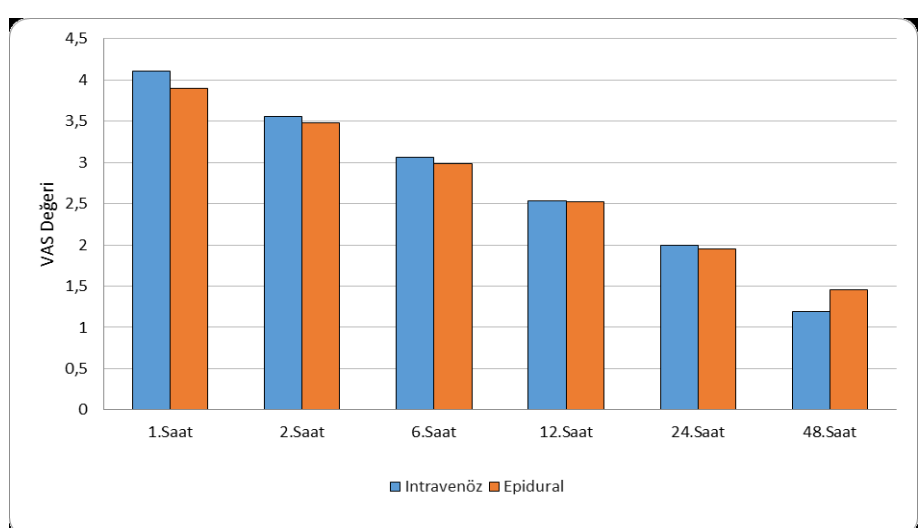

Şekil 2. Intravenöz ve epidural hasta kontrollü analjezi uygulamalarında zamana göre hastaların vizüel analog skala değerindeki değişimler.

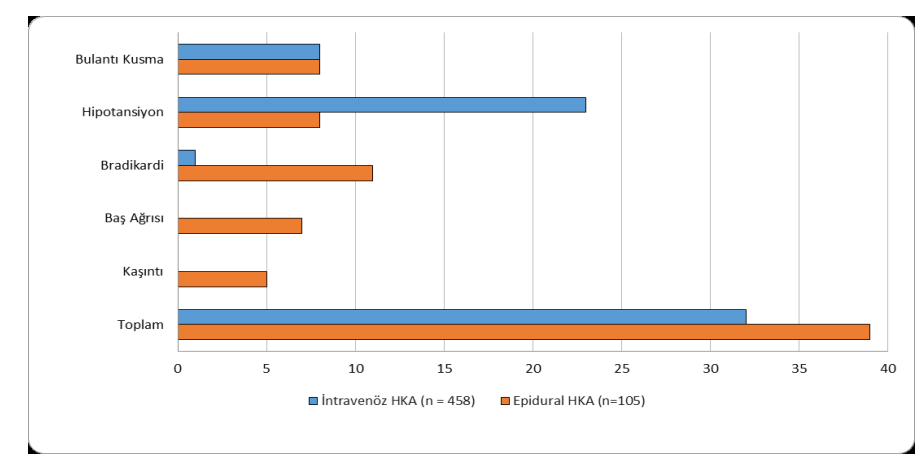

Şekil 3. Hasta kontrollü analjezi (HKA) uygulamalarında görülen yan etkiler.

TPVB + intravenöz HKA ve epidural HKA yan etki oranları TPVB + intravenöz HKA uygulanan hastalarda \%6,99, epidural HKA uygulanan hastalarda \%37,14 olarak değerlendirilmiştir. Hastalarda TPVB + intravenöz ve epidural HKA uygulama sırasında görülen yan etkiler şekil 3'te özetlenmiştir (Şekil 3).

\section{Tartışma}

Çalışmamızda iki yıllık dönemde göğüs cerrahisi merkezinde uygulanan analjezik tedavilerin sonuçları göstermiştir ki ağırlıklı olarak kliniğimizde malignite cerrahisi yapılmaktadır. $\mathrm{Bu}$ hastalarda tek enjeksiyon TPVB ve intravenöz analjezi kombinasyonu ile elastomerik infüzyonla uygulanan TEA' da sonuçların benzer olduğu görülmüştür. Komplikasyon oranları tek enjeksiyon TPVB ve intravenöz analjezi kombinasyonu yapılan grupta daha az olmakla birlikte her iki grupta da klinik olarak kabul edilebilir sınırlarda olarak bulunmuştur. Ayrıca global hasta memnuniyetleri de her iki grup için benzer oranlarda bulunmuştur.

Toraks cerrahisi en ağrılı cerrahi prosedürlerden biridir [1, 6, 8]. İyi kontrol edilemeyen postoperatif ağrı erken postoperatif komplikasyonları arttırdığı gibi kronik ağrıya bağlı uzun dönemde hastaların yaşam kalitesini ciddi anlamda olumsuz etkilemektedir $[9,10]$. Bu nedenle perioperatif ağrı yönetimi toraks cerrahisinde en önemli aşamalardan biridir. Bu amaçla intravenöz ve rejyonel yöntemlerin kombine edildiği multimodal analjezi teknikleri etkin bir analjezi sağlamaktadır $[11,12]$. Ayrıca VATS gibi daha az invaziv tekniklerin kullanılması da analjezi yönetimine ciddi katkı sağlar. Ancak VATS sonrası ağrı daha sınırlı olsa da kullanılan cerrahi aletlerin kotlar arasında oluşturduğu travmaya bağlı interkostal sinirlerde meydana gelen hasar hem ağrıyı arttırmakta hem de kronik post-torakotomi ağrısına neden olmaktadır [13]. Biz kliniğimizde hastalara TEA ya da TPVB uygulamalarıyla birlikte intravenöz analjezi uygulayarak etkin bir multimodal analjezi sağlamayı amaçlamaktayız. Postoperatif VAS skorları ve hasta 


\begin{tabular}{|c|c|c|c|c|c|c|}
\hline \multirow[t]{3}{*}{ Özellikler } & \multicolumn{3}{|c|}{$\begin{array}{c}\text { TPVB + İntravenöz } \\
\text { HKA }(n=458)\end{array}$} & \multicolumn{3}{|c|}{$\begin{array}{c}\text { Epidural } \\
\text { HKA }(n=105)\end{array}$} \\
\hline & $\mathbf{n}$ & Ort \pm SS & $\%$ & $\mathbf{n}$ & Ort \pm SS & $\%$ \\
\hline & 458 & & & 105 & & \\
\hline \multicolumn{7}{|l|}{ Cinsiyet } \\
\hline Kadın & 143 & & 31,22 & 17 & & 16,19 \\
\hline Erkek & 315 & & 68,78 & 88 & & 83,81 \\
\hline Yaş, Yıl (Ort + SS) & & $53,05 \pm 15,68$ & & & $55,34 \pm 12,59$ & \\
\hline Kadın & & $49,48 \pm 15,64$ & & & $52,65 \pm 12,13$ & \\
\hline Erkek & & $54,67 \pm 15,45$ & & & $55,86 \pm 12,68$ & \\
\hline BMI, Ort + SS & & $27,20 \pm 4,97$ & & & $26,21 \pm 4,68$ & \\
\hline Kadın & & $29,02 \pm 6,19$ & & & $28,63 \pm 5,74$ & \\
\hline Erkek & & $26,3 \pm 4,04$ & & & $25,74 \pm 4,33$ & \\
\hline Anestezi Süresi, Ort \pm SS & & $260,34 \pm 75,37$ & & & $248,62 \pm 30,73$ & \\
\hline \multicolumn{7}{|l|}{ ASA Sınıflandırılması } \\
\hline I & 1 & & 0,22 & 0 & & 0,00 \\
\hline ॥ & 161 & & 35,15 & 27 & & 25,71 \\
\hline III & 296 & & 64,63 & 78 & & 74,29 \\
\hline \multicolumn{7}{|l|}{ Tanı } \\
\hline Kitle, malignite & 345 & & 75,33 & 77 & & 73,33 \\
\hline Bronşiektazi & 16 & & 3,49 & 11 & & 10,48 \\
\hline Kist hidatik & 24 & & 5,24 & 6 & & 5,71 \\
\hline Plevral kalınlaşma, effüzyon & 44 & & 9,61 & 4 & & 3,81 \\
\hline Interstisiyel AC Hastalıkları & 5 & & 1,09 & 0 & & 0,00 \\
\hline Diğer & 24 & & 5,24 & 7 & & 6,67 \\
\hline \multicolumn{7}{|l|}{ Operasyon } \\
\hline Wedge, lobektomi, segmentektomi & 288 & & 62,88 & 74 & & 70,48 \\
\hline Pnömonektomi & 37 & & 8,08 & 15 & & 14,29 \\
\hline Eksplorasyon dekortikasyon & 49 & & 10,70 & 4 & & 3,81 \\
\hline Kistetomi & 23 & & 5,02 & 6 & & 5,71 \\
\hline Diğer & 61 & & 13,32 & 6 & & 5,71 \\
\hline \multicolumn{7}{|l|}{ Operasyon Tipi } \\
\hline VATS & 89 & & 19,43 & 0 & & 0,00 \\
\hline Torakotomi & 286 & & 62,45 & 80 & & 76,19 \\
\hline VATS + Torakotomi & 83 & & 18,12 & 25 & & 23,81 \\
\hline \multicolumn{7}{|l|}{ Hasta Memnuniyeti } \\
\hline VATS & & $2,81 \pm 0,40$ & & & 0 & \\
\hline Torakotomi & & $2,77 \pm 0,42$ & & & $2,7 \pm 0,49$ & \\
\hline VATS + Torakotomi & & $2,86 \pm 0,35$ & & & $2,76 \pm 0,44$ & \\
\hline Ek Analjezik & 25 & & 5,46 & 24 & & 22,86 \\
\hline
\end{tabular}

memnuniyeti değerlendirildiğinde uygulanan multimodal analjezinin etkili olduğunu düşünmekteyiz. Ayrıca postoperatif analjezi skorları ve hasta memnuniyet düzeyleri her iki grupta da benzer bulunmuştur. Bu durum bize torakatomi hastalarında TEA uygulamasının, VATS hastalarında da TPVB + Intravenöz analjezi uygulamasının bu gruplar için etkin bir analjezi yönetimi olduğunu düşündürmektedir.
Son yıllarda akciğer kanseri vakalarında artışla birlikte göğüs cerrahisinde malignite cerrahisi önemli bir yer işgal etmektedir. Bu hastalarda uygulanan cerrahinin daha komplike olması, hastada maligniteyebağlımevcutağrılarınolmasıvehastalıktan kaynaklanan anksiyete ağrı yönetimini bu hasta grubunda güçleştirebilir. Bu nedenle özellikle bu hasta grubunda detaylı bir preoperatif ağıı değerlendirmesi hem anksiyeteyi azaltır hem de uygulanacak 
analjezik yöntem konusunda yol gösterici olur [14, 18]. Kliniğimizde hastaların preoperatif değerlendirmesi sürecinde uygulanacak analjezik tedavi yöntemleri konusunda bilgilendirilmesinin anksiyetelerini önlemeye katkısı olduğunu düşünmekteyiz. Ayrıca çalışmamızda da malignite nedeniyle cerrahi uygulanan hasta sayısı oldukça fazlaydı ve ağırlıklı olarak torakotomi vakalarının olmasının bu durumla ilişkili olduğunu düşünmekteyiz.

Postoperatif analjezik tedavi uygulamalarında en önemli sorunlar arasında uygulanan rejyonel teknikler ve intravenöz tedaviye bağlı gelişebilen bulantı-kusma, hipotansiyon, bradikardi, kaşıntı, idrar retansiyonu ve damar sinir yaralanmaları gibi komplikasyonlardır [11]. Bu durum hem analjezik tedaviyi kesintiye uğratabilir hem de taburculuk süresini uzatabilir. Postoperatif ağrı tedavisinde altın standart olarak kabul edilen TEA'da bu komplikasyonların daha sık görülebilmesi nedeniyle TPVB gibi alternatif bloklar daha yaygın kullanılır hale gelmiştir [5]. Özellikle intravenöz analjezi ile kombine edilen TPVB' de komplikasyonlar sınırlı olduğu gibi TEA ile eşdeğer analjezi de sağlanabilmektedir [15-17]. Bizim çalışmamızda da hastalarda TPVB ile intravenöz analjezi kombinasyonunun etkin analjezi sağladığı gözlemlenmiştir.

\section{Sonuç}

Sonuç olarak toraks cerrahisi uygulanan hastalarda TPVB ve intravenöz analjezi kombinasyonu ile TEA' da benzer analjezik sonuçlar bulunmuştur. TPVB ve intravenöz analjezi kombinasyonu yapılan grupta komplikasyon oranları daha az olmakla birlikte her iki grupta da klinik olarak kabul edilebilir sınırlarda yan etki oranları gözlenmiştir. Analjezi protokollerinin ve algoritmaların klinisyenlerin deneyimi ve uygulanan cerrahi işleme göre belirlenmesinin etkin analjezi sağlayacağını düşünmekteyiz.

\section{Çalışma Sınırlamaları}

Çalışmamızın kısıtlııkları, retrospektif tasarımı olması ve tek merkezli bir çalışma olmasıdır. Uygulanan analjezik yöntemlerin veri eksikliği nedeniyle uzun dönem kronik ağrı gelişimi ile ilişkisi değerlendirilememiştir.

\section{Çıkar Çatışması/Finansal Destek Beyanı}

Çalışmayı maddi olarak destekleyen kişi/kuruluş yoktur ve yazarların herhangi bir çıkara dayalı ilişkisi yoktur

\section{Kaynaklar}

1. Marshall K, McLaughlin K. Pain Management in Thoracic Surgery. Thorac Surg Clin. 2020; 30: 339-46

2. Liu X, Song $T$, Xu HY, Chen X, Yin P, Zhang et al. The serratus anterior plane block for analgesia after thoracic surgery: A metaanalysis of randomized controlled trails. Medicine (Baltimore). 2020; 99: 20286

3. Razi SS, Stephens-McDonnough JA, Haq S, et al. Significant reduction of postoperative pain and opioid analgesics requirement with an Enhanced Recovery After Thoracic Surgery protocol. J Thorac Cardiovasc Surg. 2021; 161: 1689-701

4. Fang B, Wang Z, Huang X. Ultrasound-guided preoperative single-dose erector spinae plane block provides comparable analgesia to thoracic paravertebral block following thoracotomy: a single center randomized controlled double-blind study. Ann Transl Med. $2019 ; 7: 174$

5. Zengin M, Baldemir R, Ulger G, et al. Postoperative Analgesic Efficacy of Thoracic Paravertebral Block and Erector Spinae Plane Block Combination in Video-Assisted Thoracic Surgery. Cureus. 2021; 13: 15614

6. Luketich JD, Land SR, Sullivan EA, et al. Thoracic epidural versus intercostal nerve catheter plus patient-controlled analgesia: a randomized study. Ann Thorac Surg. 2005; 79: 1845-9

7. Nagaraja PS, Ragavendran S, Singh NG, et al. Comparison of continuous thoracic epidural analgesia with bilateral erector spinae plane block for perioperative pain management in cardiac surgery. Ann Card Anaesth. 2018; 21: 323-27.

8. American Society of Anesthesiologists Task Force on Acute Pain Management. Practice guidelines for acute pain management in the perioperative setting: an updated report by the American Society of Anesthesiologists Task Force on Acute Pain Management. Anesthesiology. $2012 ; 116: 248-73$

9. Ochroch EA, Gottschalk A. Impact of acute pain and its management for thoracic surgical patients. Thorac Surg Clin. 2005; 15: 105-21.

10. Rogers ML, Henderson L, Mahajan RP, et al. Preliminary findings in the neurophysiological assessment of intercostal nerve injury during thoracotomy. Eur J Cardiothorac Surg. 2002 ; 21: 298-301 
11. Sugiyama T, Kataoka Y, Shindo K, et al. Retrolaminar Block Versus Paravertebral Block for Pain Relief After Less-Invasive Lung Surgery: A Randomized, Non-Inferiority Controlled Trial. Cureus. 2021; 13: 13597

12. Piccioni F, Segat $M$, Falini $S$, et al. Enhanced recovery pathways in thoracic surgery from Italian VATS Group: perioperative analgesia protocols. J Thorac Dis. 2018; 10: 555-63

13. Hirai K, Usuda J. Uniportal video-assisted thoracic surgery reduced the occurrence of post-thoracotomy pain syndrome after lobectomy for lung cancer. J Thorac Dis. 2019; 11: 3896-902

14. Shady RHA, Asmaa IAS, Marwa FM. Effectiveness of acupressure in the reduction of pain and anxiety among patients with open thoracotomy. American Journal of Nursing. 2020; 8: 182-91

15. Karmakar MK. Thoracic paravertebral block. Anesthesiology. 2001; 95: 771-80
16. Yeung JH, Gates S, Naidu BV, et al. Paravertebral block versus thoracic epidural for patients undergoing thoracotomy. Cochrane Database Syst Rev. 2016; 2: 9121

17. Kotzé A, Scally A, Howell S. Efficacy and safety of different techniques of paravertebral block for analgesia after thoracotomy: a systematic review and metaregression. $\mathrm{Br} J$ Anaesth. 2009 ; 103: 626-36

18. Alagöz A, Ergüven $M$, Tunç $M$, et al. Preoperatif Anksiyete Skoru İle Torakotomi Sonrası Ağrı Arasında Bir Korelasyon Var mıdır?. Ortadogu Medical Journal/Ortadoğu Tıp Dergisi 2012; 4: 117-21. 